\title{
Primary Retroperitoneal Synovial Sarcoma
}

\section{: A Case Report}

\begin{abstract}
A case of a 36-yr-old woman with retroperitoneal synovial sarcoma is described. Her presenting symptom was epigastric pain that radiating to the back. On radiologic study, bulky retropancreatic soft tissue mass was detected which showed cystic and solid components. At operation, complete resection of the tumor was not possible because of the adhesion to the vena cava and the liver. During the follow-up, extensive tumor recurrence and liver metastasis were revealed. Primary retroperitoneal synovial sarcoma is a very rare malignant tumor with high mortality and recurrence rates. Retroperitoneal synovial sarcoma usually appears as a nonspecific soft tissue mass that do not have specific imaging features differentiating it from other mesenchymal tumors. However general radiologic findings and anatomic location of the tumor may help the diagnosis. In addition, synovial sarcoma should be included in the differential diagnosis of retroperitoneal soft tissue mass detected in young adults.
\end{abstract}

Key Words : Tomography, X-ray Computed; Retroperitoneal Neoplasms; Sarcoma, Synovial

\author{
Hotaek Song, Byung Hee Koh, \\ On Koo Cho, Hyunchul Rhim, Yongsoo Kim, \\ Eun Kyung Hong ${ }^{*}$, Yong Wook Park ${ }^{\dagger}$ \\ Department of Diagnostic Radiology, College of \\ Medicine, Hanyang University; Department of \\ Pathology ${ }^{*}$, National Cancer Center, Koyang; \\ Department of Pathology ${ }^{\dagger}$, College of Medicine, \\ Hanyang University, Seoul, Korea \\ Received : 2 March 2001 \\ Accepted : 3 August 2001 \\ Address for correspondence \\ Byung Hee Koh, M.D. \\ Department of Diagnostic Radiology, Hanyang \\ University Hospital, 17 Haengdang-dong, \\ Sungdong-gu, Seoul 133-792, Korea \\ Tel : +82.2-2290-9159, Fax : +82.2-2293-2111 \\ E-mail : bhkoh@hanyang.ac.kr
}

\section{INTRODUCTION}

The term synovial sarcoma refers to the morphology that resembles developing synovium (1). Eighty five to $95 \%$ of synovial sarcomas occur in the extremities near the large joints. Only $5-15 \%$ of synovial sarcomas affect the head and neck, mediastinum, abdominal wall, and retroperitoneum (1-4). Primary retroperitoneal synovial sarcoma is extremely rare and has poor prognosis (6). There are 19 cases of retroperitoneal synovial sarcomain the English literature.

\section{CASE REPORT}

A 36-yr-old woman was admitted to our hospital with epigastric pain radiating to the back, which had persisted for 1 week. On physical examination there was tenderness in the epigastric region. The pulse rate was 74 per minute and blood pressure was 110/80 mmH g. Complete blood cell count reveal ed hemoglobin $8.7 \mathrm{~g} / \mathrm{dL}$ with mean corpuscular volume of $76.5 \mathrm{fL}$ and white blood count 6,700 cells $\mathrm{s}_{\mathrm{k}} \mathrm{L}$ with $73.6 \%$ of polymorphonuclear leukocytes.

Ultrasonogram showed a solid and cystic retropancreatic mass (Fig. 1A). Computed tomography (CT) showed a 10$\mathrm{cm}$ low attenuating retropancreatic mass with a mild contrast enhancement. It extended from the portocaval space of the hepatic hilum to just above the right renal vein. The margin of themass was relatively well-defined with surrounding structures by the fat plain but the border between the mass and the vena cava was somewhat blurred (Fig. 1B). Duodenography showed a widening of the duodenal C-loop by the mass but there was no passage disturbance (Fig. 1C). Celiac arteriography showed avascular mass, which displaced the surrounding vessels without tumor staining.

At operation, the tumor adhered to the inferior vena cava and caudate lobe of the liver. Gross inspection of the resected specimen reveal ed an 8-cm lobulated mass in dark brownish color. The cut surface of the mass showed a grayish tan, fish flesh appearance with hemorrhage (Fig. 1D).

$\mathrm{H}$ istologic examination demonstrated a monophasic fibrous pattern consisting of closely packed round to oval spindle shaped cells with hyperchromatic nuclei and small amount of eosinophilic cytoplasm without evidence of epithelial differentiation (Fig. 1E). Immunohistochemically the tumor cells showed strong positivity for vimentin and focally weak positivity for cytokeratin (Fig. 1F), but were negative for epithelial membrane antigen and S-100 protein. Mitoses were frequently seen. Final diagnosis was a poorly differentiated primary retroperitoneal synovial sarcoma.

Five months from the operation, she was readmitted due to low back pain and jaundice. Follow-up CT scan showed extensive tumor recurrence at the original tumor bed as well as liver metastasis (Fig. 1G). 

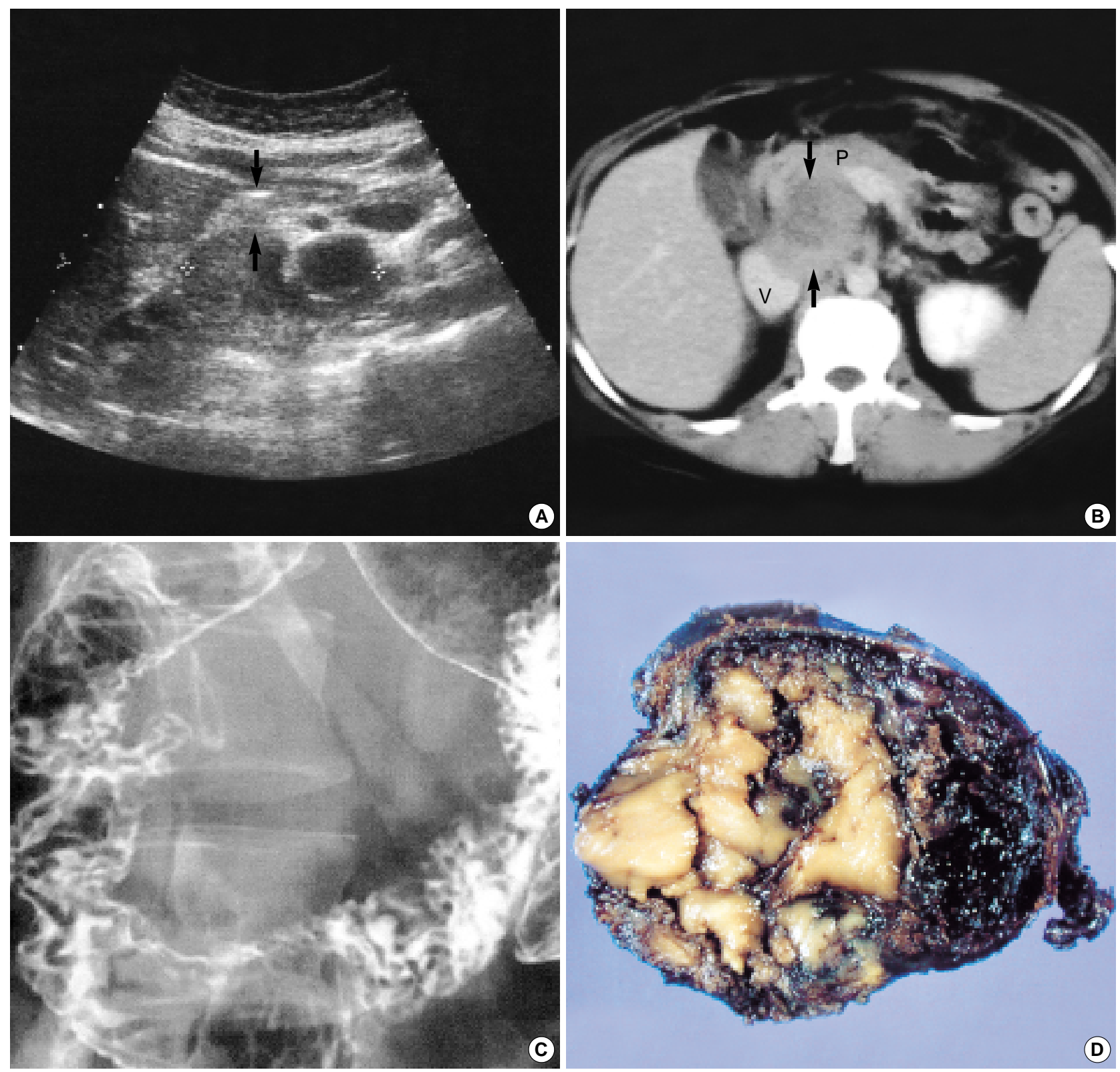

Fig. 1. A 36-yr-old woman with primary retroperitoneal synovial sarcoma. (A) Transverse scan of ultrasonogram at the level of celiac axis shows a lobulated solid and cystic mass displacing the pancreas (anteriorly arrows). (B) Delayed contrast-enhanced CT scan at the level of celiac axis shows a low-attenuating mass (arrows) with anterior displacement of pancreas ( $p$ ) and lateral displacement vena cava (v). Obliteration of fat plane between mass and vena cava is noted. (C) Duodenography shows widening of duodenal C-loop. (D) The cut surface of the lobulated mass shows a grayish tan, fish flesh appearance with hemorrhage (Fig. 1 continued next).

\section{DISCUSSION}

The exact nature of differentiation in synovial sarcoma is controversial. Potential origins of the synovial sarcomaindude normal synovium, arthrogenous mesenchyma, and primitive pluripotent mesenchyma. Usually synovial sarcoma occurs in the vicinity of the joint capsules, bursae, and tendon sheaths. But the occurrences of the tumor in various extraarticular sites such as the mediastinum, abdominal wall, retroperito- neum, intraperitoneum, and esophagus have been reported (2-4). Synovial sarcoma is most prevalent in adolescents and young adults between 15 and $40 \mathrm{yr}$ of age.

A primary retroperitoneal sarcoma has been defined as a tumor arising in the retroperitoneal space with an origin of me sodermal structures exclusive of bony, renal, visceral, adrenal, and pancreatic tissues (6).

Retroperitoneal synovial sarcoma usually appears as a nonspecific soft tissue mass that does not have specific imaging 

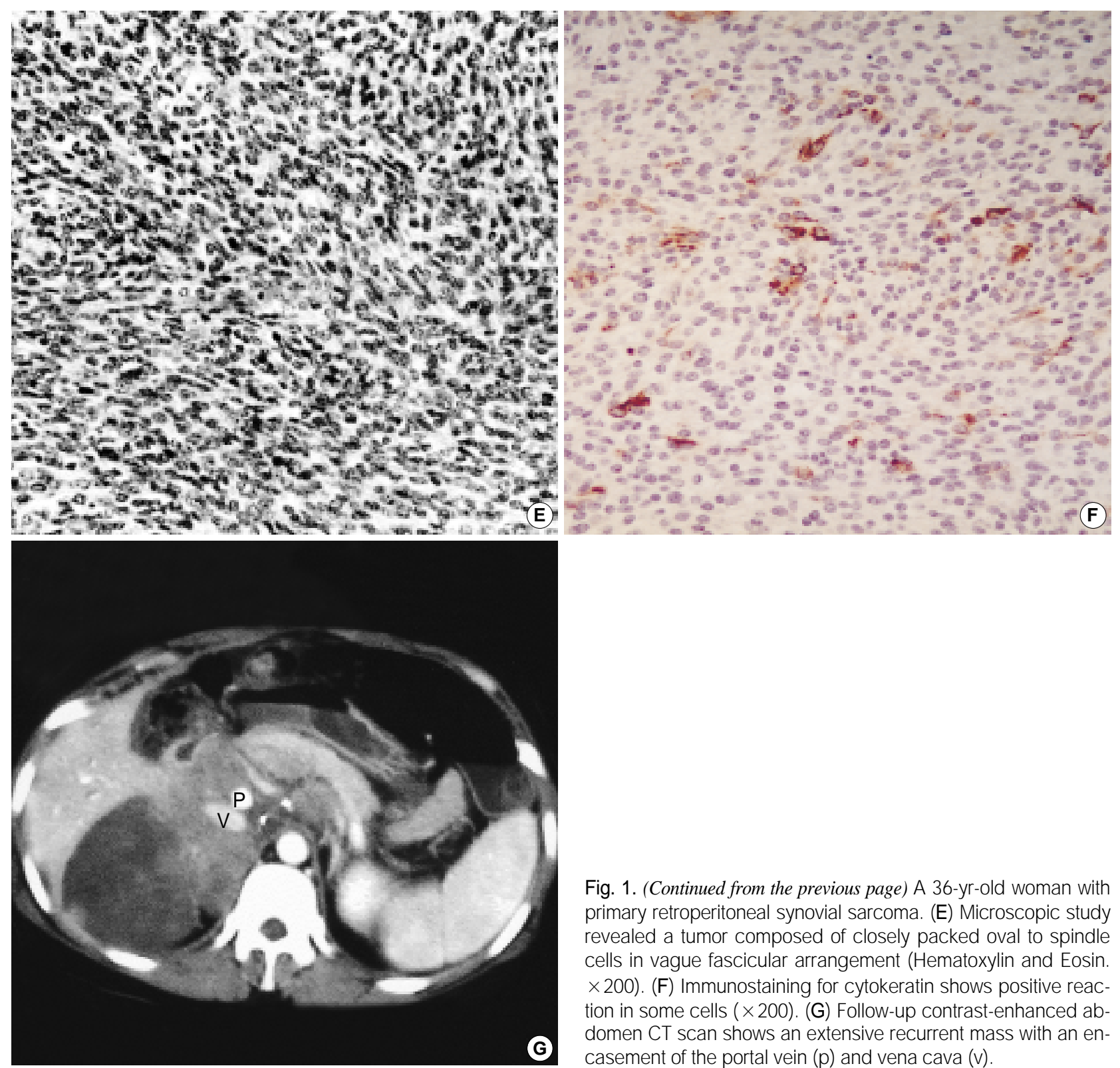

Fig. 1. (Continued from the previous page) A 36-yr-old woman with primary retroperitoneal synovial sarcoma. (E) Microscopic study revealed a tumor composed of closely packed oval to spindle cells in vague fascicular arrangement (Hematoxylin and Eosin. $\times 200$ ). (F) Immunostaining for cytokeratin shows positive reaction in some cells $(\times 200)$. (G) Follow-up contrast-enhanced abdomen CT scan shows an extensive recurrent mass with an encasement of the portal vein ( $p)$ and vena cava (v).

features differentiating it from other mesenchymal tumors $(2,3,8)$. H owever radiologic findings and anatomic location of the tumor may help the diagnosis. In addition, synovial sarcoma should be considered when retroperitoneal soft tissue mass is found in young adults. On CT, these tumors are hypo-dense and may show an irregular enhancement in the periphery with a poor enhancement in the central area, reflecting the necrotic, cystic, and hemorrhagic changes (8). In the region of 30\% of cases show intratumoral cal cification and extensive cal cification suggest a favorable prognosis $(3,8)$. CT is still recommended as the best imaging method for assessing the local extent of the primary tumor and is a useful tool in the planning of appropriate therapy as well as evaluation of tumor response to ongoing treatment (8).

$\mathrm{H}$ istologically there are two types of synovial sarcomas: biphasic and monophasic. Biphasic type is an admixture of epithelial cells and spindle cells. M onophasic type is composed of either only epithelial cells or spindle cells (1-4). Both types have a mortality rate of $40 \%$. The known poor prognostic factors are frequent mitotic figures (morethan 10 or 15 mitoses per $10 \mathrm{HPF}$ ) and extensive tumor necrosis, and least favorable for the poorly differentiated (small cell) type. Favorable factors are young age of the patient ( $15 \mathrm{yr}$ or younger) and tumor size smaller than $5 \mathrm{~cm}$, and distal rather than proximal location in the extremities (5). Pathologically, major differential diagnosis of monophasic fibrous synovial sarcoma includes hemangiopericytoma, malignant peripheral nerve sheath tumor (MPN ST, malignant schwannoma), and fibro- 
sarcoma. Hemangiopericytoma shows abundant vasculature, with no spindle cell and negativity for cytokeratin. MPN ST may show cl ose resemblance to the monophasic fibrous type of synovial sarcoma, but there is no evidence that synovial sarcoma arises in a large nerve or in a patient with neurofibromatosis. Immunohistochemically MPN ST shows focal weak positivity for S-100 and negativity for cytokeratin. Fibrosarcoma composed of long spindle cells and shows arrange ment of the fibroblasts in distinct intersecting fascicles (herringbone pattern) (7).

Surgical ablation remains the mainstay of management of retroperitoneal sarcomas, but complete resection rate of is approximately $50 \%$ (6). The recurrence rate ranged from $28 \%$ to $36 \%$ even with adequate surgical and adjunctive therapies (3).

\section{REFERENCES}

1. Cotran RS, Kumar V, Robbins SL. Pathologic basis of disease, 5th ed.
Philadelphia: W.B. Saunders 1994: 1261-9.

2. Shmookler BM. Retroperitoneal synovial sarcoma. A report of four cases. Am J Clin Pathol 1982; 77: 669-73.

3. Ko SF, Chou FF, Huang CH, Ng SH, Wan YL, Lee TY, Lin JW, Chen WJ. Primary synovial sarcoma of the gastrocolic ligament. Br J Radiol 1998; 71: 438-40.

4. Fetsch JF, Meis JM. Synovial sarcoma of the abdominal wall. Cancer 1993; 72: 469-77.

5. Kransdort MJ. Malignant soft-tissue tumors in a large referral population: distribution of diagnoses by age, sex, and location. Am J Roentgenol 1995; 164: 129-34.

6. Ziarn BH, Makley JT, Carter JR. Primary retroperitoneal sarcomas: common symptoms, common diagnoses, uncommon disease. Clin Orthop 1996; 331: 277-82.

7. Enzinger FM, Weiss SW. Soft tissue tumors, 3rd ed. St. Louis: Mosby 1995: 759-86.

8. Israels SJ, Chan HS, Daneman A, Weitzman SS. Synovial sarcoma in childhood. Am J Roentgenol 1984; 142: 803-6. 